\title{
Time-dependent photoionization and fluorescence line emission in gamma-ray burst environments
}

\author{
M. Böttcher ${ }^{1}$, C.D. Dermer ${ }^{2}$, and E.P. Liang ${ }^{1}$ \\ 1 Department of Space Physics and Astronomy, Rice University, 6100 S. Main St., Houston, TX 77005-1892, U.S.A. \\ 2 E. O. Hulburt Center for Space Research, Code 7653, Naval Research Laboratory, Washington, DC 20375-5352, U.S.A.
}

Received January 21; accepted April 16, 1999

\begin{abstract}
If $\gamma$-ray bursts (GRBs) are associated with dense star-forming regions, photoelectric absorption by the circumburster material (CBM) will occur. As the burst evolves, the surrounding material is photoionized, leading to fluorescence line emission and reduced photoelectric absorption opacity. We have numerically simulated this problem, accounting for all relevant microscopic processes. We find that if GRBs are hosted in a quasi-isotropic surrounding medium, photoionization of the CBM leads to a constant but weak level of delayed fluorescence line emission on timescales of weeks to months after the GRB. A temporally evolving $\mathrm{Fe} \mathrm{K}$ edge absorption feature can serve as diagnostic tool for redshift measurements.
\end{abstract}

Key words: atomic processes — radiative transfer gamma-rays: bursts - X-rays: bursts

\section{Introduction}

If GRBs are associated with the collapse of massive stellar objects (e.g., Paczyński 1998; Woosley 1993), they are expected to be associated with star-forming regions. If this is true, photoelectric absorption and fluorescence line emission in the vicinity of GRBs might lead to observable consequences such as absorption edges and emission line features. It has been suggested that absorption edges as well as fluorescence lines and resonance-scattered burst emission could be detectable with rapid (Mészáros \& Rees 1998) or long-term (Ghisellini et al. 1998) follow-up observations of GRB afterglows and thus provide a possibility for direct GRB redshift measurements. The process of time-dependent photoionization and photoelectric absorption has been studied by Perna \& Loeb (1998). They focus on optical absorption lines from the CBM and suggest that temporal evolution of absorption line shapes and equivalent widths can be used to map the CBM. In a recent paper (Böttcher et al. 1999), we adopted a similar approach to the one used by Perna \& Loeb (1998), but additionally implemented the Auger process and fluorescence line emission following inner-shell ionization events,

Send offprint requests to: M. Böttcher and used a realistic representation of the temporal evolution of the GRB radiation. Here we give a short summary of the main results of this analysis, focusing on observable effects in the X-ray regime.

\section{Model assumptions}

We simulate a relativistic blast wave expanding into and photoionizing a stationary external medium. The evolution of the blast wave and of the GRB spectrum is represented by the analytic parametrization of Dermer et al. (1999). The model is mainly determined by the total explosion energy, $E_{0}=10^{54} E_{54} \mathrm{erg}$, the initial bulk Lorentz factor $\Gamma_{0}$, and the index $g$ characterizing the radiative regime of the blast wave evolution, with $g=3 / 2$ corresponding to a non-radiative (adiabatic) blast wave, while $g=3$ describes a fully radiative blast wave. The CBM density distribution $n_{\text {ext }}(r)=n_{0}\left(r / r_{\mathrm{d}}\right)^{-\eta}$ is assumed to have a power-law profile in distance from the burst source determined by the density $n_{0}$ at the deceleration radius $r_{\mathrm{d}}$ and the power-law index $\eta$.

We solve the time-dependent radiation transfer and photoionization problem for $\mathrm{H}, \mathrm{He}, \mathrm{C}, \mathrm{N}, \mathrm{O}, \mathrm{Ne}, \mathrm{Mg}, \mathrm{Si}, \mathrm{S}$, $\mathrm{Ar}, \mathrm{Ca}, \mathrm{Fe}$, and Ni. For the results presented in this paper we assume standard solar-system element abundances. We use the photoionization cross sections for all subshells of all elements using the relevant subroutines of the XSTAR code (Kallman \& Krolik 1998). Auger and radiative transitions following inner-shell photoionization events are calculated using the tables of Kaastra \& Mewe (1993). We account for the light-travel time delay of fluorescence line emission. Since we assume densities of $n_{0} \lesssim 10^{6} \mathrm{~cm}^{-3}$, recombination is negligible.

\section{Results}

We have carried out a large number of simulations using different assumptions on the burst spectral properties and on the density and radial extent of the CBM. The two effects that are potentially relevant to observations are discussed in the following two subsections. 


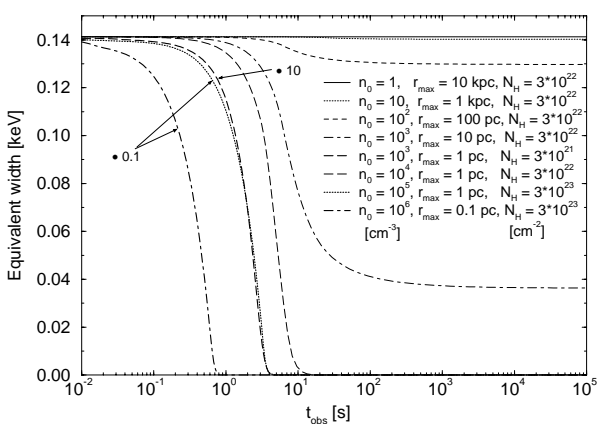

Fig. 1. Time dependence of the equivalent width of the Fe K absorption edge for a variety of CBM parameters. For the thin curves, the hydrogen column density is held constant at $N_{\mathrm{H}}=310^{22} \mathrm{~cm}^{-2}$. For heavy curves, different values of $N_{\mathrm{H}}$ are used

\subsection{The Iron $K$ absorption edge}

Our simulations indicate that an iron absorption edge could be observable. However, in order to attribute the absorption unambiguously to the CBM - rather than to foreground absorption - a decrease of the K-edge opacity, caused by photoionization, must be observed. In Fig. 1, the time dependence of the $\mathrm{Fe} \mathrm{K} \alpha$ edge for various assumptions on the CBM density and extent is plotted. The figure demonstrates that bright bursts $\left(E_{54} \sim 1\right)$ can ionize star-forming regions completely, leading to the required significant decrease of the Fe $\mathrm{K}$ edge opacity. However, if absorption features vary with time due to photoelectric absorption, this can only occur on $\lesssim$ minute time scales. This implies that prompt GRB observations with moderate energy resolution at a few $\mathrm{keV}$ are required in order to detect these varying absorption features. The absorption features vary on the timescale of the burst duration because most of the fluence of the burst is emitted during the prompt burst phase. This is in contradiction to the assumption of Perna \& Loeb (1998) who used a slower decay of the X-ray afterglow, $F_{\nu}(t) \propto t^{-3 / 4}$, inconsistent with observations.

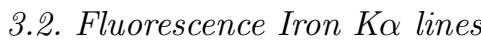

The iron $\mathrm{K} \alpha$ fluorescence line flux increases on the burst duration time scale and remains at a roughly constant level over the light-travel time through the CBM. This implies that the Fe $\mathrm{K} \alpha$ line emission dominates the flux at $6.4-6.7 \mathrm{keV}$ (in the GRB's rest frame) after the continuum flux in this energy range has decayed below the line flux level. In Fig. 2 the maximum $\mathrm{Fe} \mathrm{K} \alpha$ line luminosities and corresponding fluxes for a burst located at $z=1$ for a variety of CBM parameters with constant $N_{\mathrm{H}}=310^{22} \mathrm{~cm}^{-2}$ are plotted. We find that the fluorescence line luminosities are generally low, $L_{\mathrm{FeK} \alpha} \lesssim 10^{41} \mathrm{erg} \mathrm{s}^{-1}$, corresponding to $F_{\mathrm{FeK} \alpha}(z=1) \lesssim 10^{-16} \mathrm{erg} \mathrm{cm}^{-2} \mathrm{~s}^{-1}$ for standard solar-system metal abundances and a quasi-isotropic

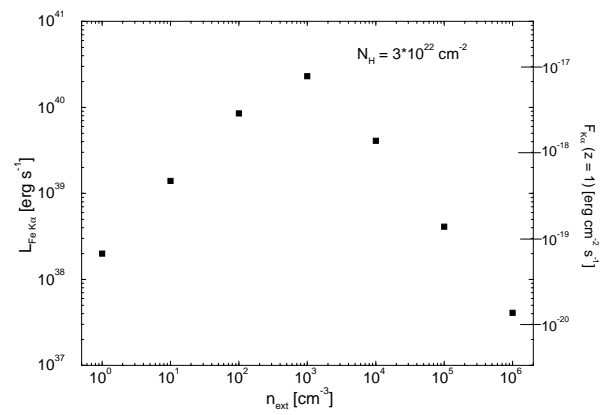

Fig. 2. The maximum line luminosities in the $\mathrm{Fe} \mathrm{K} \alpha$ lines for different CBM parameters. The hydrogen column is held constant at $N_{\mathrm{H}}=310^{22} \mathrm{~cm}^{-2}$. The right scale indicates the corresponding line fluxes if the burst is located at $z=1$

CBM. $L_{\mathrm{FeK} \alpha}$ increases with increasing $N_{\mathrm{H}}$. However, there is a limit of $N_{\mathrm{H}} \ll 10^{24} \mathrm{~cm}^{-2}$ since for higher values Thomson scattering effects on the hard X-ray radiation would become observable. In particular, short-timescale variability would be smeared out over the typical photon escape timescale $t_{\mathrm{esc}} \sim r_{\max } \tau_{\mathrm{T}} / c$ if $\tau_{\mathrm{T}} \gtrsim 1$, in contrast to the observed $\lesssim 1 \mathrm{~ms}$ variability in some GRB pulses. In Fig. 2, the line luminosities increase with density for small densities since in this limit the entire CBM is ionized, and an incrasing density leads to an increasing amount of material contributing to the fluorescence line emission. In the high-density limit, an increasing density leads to a decrease of the ionization radius and thus to a decrease of the fluorescence-line emitting volume. Our results indicate that if the $\mathrm{CBM}$ is quasi-isotropic and metals have abundances close to the solar-system values, fluorescence lines will hardly be detectable even with future X-ray telescopes (AXAF, XMM, Astro-E). The recent marginal detections of the $\mathrm{Fe} \mathrm{K} \alpha$ fluorescence line in the X-ray afterglows of GRB 970508 (Piro et al. 1998) and GRB 980828 (Yoshida et al. 1998) can not have originated in an isotropic medium as investigated in this paper.

Acknowledgements. We wish to thank Jon C. Weisheit and Tim Kallman for helpful discussions. This work was partially supported by NASA grant NAG 5-4055.

\section{References}

Böttcher M., et al., 1999, A\&A (in press) (astro-ph/9809156) Dermer C.D., Chiang J., Böttcher M., 1999, ApJ 513 (in press) Ghisellini G., Haardt F., Campana S., et al., 1998, ApJ (submitted) (astro-ph/9808156)

Kaastra J.S., Mewe R., 1993, A\&AS 97, 443

Kallman T.R., Krolik J.H., 1998, The XSTAR User's Guide

Mészáros P., Rees M.J., 1998, MNRAS 299, L10

Paczyński B., 1998, ApJ 494, L45

Perna P., Loeb A., 1998, ApJ 501, 467

Piro L., et al., 1998 (these proceedings)

Yoshida A., et al., 1998 (these proceedings) 